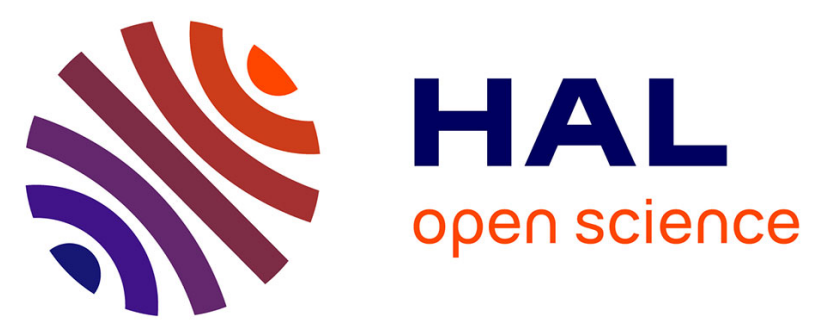

\title{
Content based Medical Image Retrieval: use of Generalized Gaussian Density to model BEMD's IMF.
}

Said Jai Andaloussi, Mathieu Lamard, Guy Cazuguel, Hamid Tairi, Mohamed Meknassi, Béatrice Cochener, Christian Roux

\section{- To cite this version:}

Said Jai Andaloussi, Mathieu Lamard, Guy Cazuguel, Hamid Tairi, Mohamed Meknassi, et al.. Content based Medical Image Retrieval: use of Generalized Gaussian Density to model BEMD's IMF.. World Congress on Medical Physics and Biomedical Engineering, Sep 2009, Munich, Germany. hal00424462

\section{HAL Id: hal-00424462 \\ https://hal.science/hal-00424462}

Submitted on 15 Oct 2009

HAL is a multi-disciplinary open access archive for the deposit and dissemination of scientific research documents, whether they are published or not. The documents may come from teaching and research institutions in France or abroad, or from public or private research centers.
L'archive ouverte pluridisciplinaire HAL, est destinée au dépôt et à la diffusion de documents scientifiques de niveau recherche, publiés ou non, émanant des établissements d'enseignement et de recherche français ou étrangers, des laboratoires publics ou privés. 


\title{
Content based Medical Image Retrieval: use of Generalized Gaussian Density to model BEMD's IMF.
}

\author{
${ }^{1,2,4}$ Said Jai-Andaloussi, ${ }^{3,4}$ Mathieu Lamard, ${ }^{1,4}$ Guy Cazuguel, ${ }^{2}$ Hamid Tairi, \\ ${ }^{2}$ Mohamed Meknassi, ${ }^{3,4,5}$ Béatrice Cochener, ${ }^{1,4}$ Christian Roux \\ ${ }^{1}$ INSTITUT TELECOM; TELECOM Bretagne; UEB; Dpt ITI, Brest, F-29200, France; \\ ${ }^{2}$ Département de mathématique et informatique faculté des sciences, Fès 30000, Maroc; \\ ${ }^{3}$ Univ Bretagne Occidentale, Brest, F-29200 France; \\ ${ }^{4}$ Inserm, LaTIM U650, IFR 148 ScInBioS - Science et Ingénierie en Biologie-Santé, BREST, F-29200 FRANCE; \\ ${ }^{5}$ CHU de Brest, Service d'Ophtalmologie, Brest, F-29200 France
}

\begin{abstract}
In this paper, we address the problem of medical diagnosis aid through content based image retrieval methods. We propose to characterize images without extracting local features, by using global information extracted from the image Bidimensional Empirical Mode Decomposition (BEMD). This method decompose image into a set of functions named Intrinsic Mode Functions (IMF) and a residu. The generalized Gaussian density function (GGD) is used to represent the coefficients derived from each IMF, and the Kullback-Leibler Distance (KLD) compute the similarity between GGDs. Retrieval efficiency is given for different databases including a diabetic retinopathy, and a face database. Results are promising: the retrieval efficiency is higher than $85 \%$ for some cases.
\end{abstract}

Keywords - Content-Based Image Retrieval, BEMD, Generalized Gaussian density, Kullback-Leibler distance.

\section{INTRODUCTION}

Nowadays, medical systems produce a great amount of data images. Fast access to such huge databasse requires efficient indexing algorithms. CBIR system (Content-Based Image Retrieval) is one of the possible solutions to effectively manage image databases [1], [2]. The purpose is to retrieve desired images from databases using only the numerical content of images. Typical CBIR systems can be decomposed in two steps: at first, extraction of features from each medical image in the image database; then, the feature vector of a query image is computed and compared to the images feature vectors of the database. For definition and extraction of visual features, many methods have been proposed, includind image segmentation and image characterization using wavelet transform and gabor filter bank [2, $3,4,5,6]$,

Recently, a novel multiresolution decomposition method, the Empirical Mode Decomposition (EMD), was introduced by Huang and al [7]. The EMD is an adaptive decomposition which decompose any signal into a redundant set of signals denoted IMF and a residue. The original signal may be reconstruct by adding all the IMFs together and the residue, without information loss or distortion. EMD does not use any pre-determined filter or basis functions, which is quite different from Fourier analysis and wavelet analysis. In 2003, Nunes and al. [8] extend the 1DEMD signal analysis to bidimensional signals and specifically to image processing (Bidimensional Empirical Mode Decomposition : BEMD). We propose in this work a new medical retrieval method which takes advantage of image BEMD. Wouwer and al. [9] used generalized Gaussian density functions (GGD functions) to represent texture images in the wavelet domain. In this paper we propose a new algorithm, based on Wouwer work, using BEMD decomposition instead of wavelet transform.

The setup of the paper is as follows. Section II-A describes the database we used for evaluation. Section II-B describes the BEMD method. Section II-C explains how to use GGD functions to represent the coefficients derived from BEMD. The retrieval method and Kullback-Leibler distance are described in section II-D and results are given in section III. We end with a discussion and conclusion in section IV.

\section{MATERIAL AND METHODS}

\section{A. Databases}

Two databases are used to test our method.

1) Diabetic retinopathy database: the diabetic retinopathy database, developed specifically in our team, contains retinal images of diabetic patients, with associated contextual anonymized information on the pathology. The database is made up of 63 patient files, containing 1045 photographs alltogether, and is divided in 6 levels of disease severity (6 classes). Images have a definition of 1280 pixels/line for 1008 lines/image. The patients were seen in consultation in Brest university hospital (France), within the screening or the follow-up of diabetic patient. The images were acquired 
by experts using a Topcon Retinal Digital Camera (TRC50IA) connected to a computer. An example of image series is given in Fig 1.
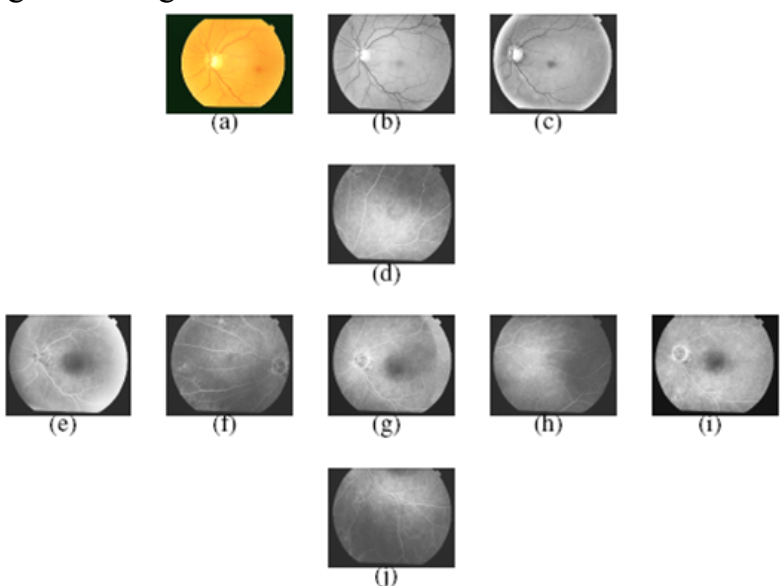

Fig. 1 Photograph series of a patient eye

2) FaceDataBase: This database [10] is used as a benchmark database. There are ten different images of each of 40 distinct subjects. For some subjects, the images were taken at different times, varying the lighting, facial expressions (open / closed eyes, smiling / not smiling) and facial details (glasses / no glasses). All the images were taken against a dark homogeneous background with the subjects in an upright, frontal position (with tolerance for some side movement). Images have a resolution of 92x112 pixels; each pixel is coded on a byte.

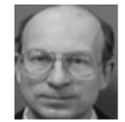

(a)

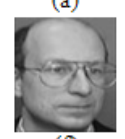

(f)

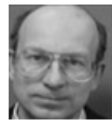

(b)

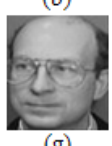

(g)

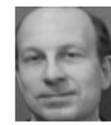

(c)

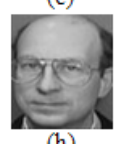

(h)

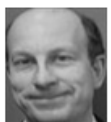

(d)

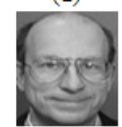

(i)

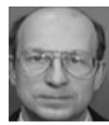

(e)

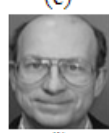

(j)
Fig. 2 Ten images sequence of the same person's face

\section{B. BEMD}

The EMD method is an adaptive decomposition which allows to decompose any signal into a redundant set of signals denoted IMF and a residue. These IMFs are obtained by means of an algorithm called sifting process.

Given a signal $s(t)$, the sifting process of EMD can be summarized as follows.

1. Initialise: $r_{0}=s$ (the residual) and $j=1$ (index number of IMF),

2. Extract the $j$ th IMF:

3. (a) Initialise $h_{0}=r_{j-1} ; i=1$;

(b) Extract local minima/maxima of $h_{i-1}$; (c) Compute upper envelope and lower envelope funtions $x_{i-1}$ and $y_{i-1}$ by interpolating, respectively, local minima and local maxima of $h_{i-1}$;

(d) Compute $m_{i-1}=\left(x_{i-1}+y_{i-1}\right) / 2$ (mean envelope),

(e) Update $h_{i}=h_{i-1}-m_{i-1}$ and $i=i+1$;

(f) Calculate stopping criterion (standard deviation $\left.S D_{j i}\right)$

(g) Repeat steps (b) to (f) until $S D_{j i} \leq S D M A X$ and put then $s_{j}=h_{i}(j t h$ IMF)

4. Update residual $r_{j}=r_{j-1}-s_{j}$;

5. Repeat steps $2-4$ with $j=j+1$ until the number of extrema in $r_{j}$ is less than 2.

After IMFs are extracted through the sifting process, the original signal $s(t)$ can be represented like this:

$$
s(t)=\sum_{j=1}^{\mathrm{n}} \operatorname{Imf}_{\mathrm{j}}(\mathrm{t})+r(t)
$$

The Standard Deviation (SD) is the criteria to stop sifting process, computed from two consecutive sifting.

$$
\mathrm{SD}_{\mathrm{ij}}^{2}=\sum_{k=1}^{\mathrm{K}}\left[\frac{\left|\left(h_{j(i-1)}(k)-h_{j i}(k)\right)\right|^{2}}{h_{j(i-1)}^{2}(k)}\right]
$$

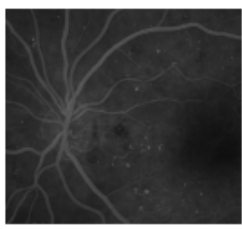

Original image
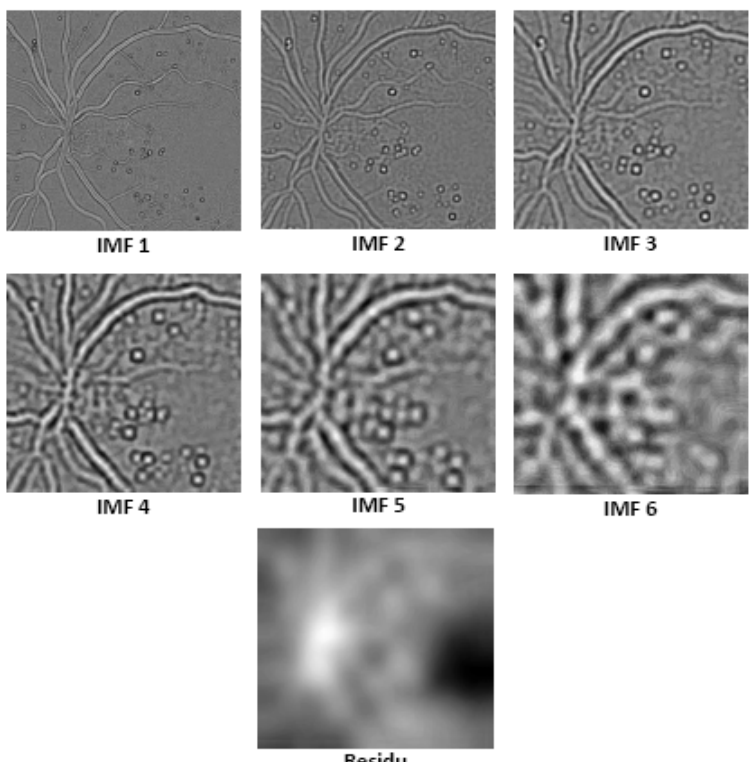

IMF 6

IMF 4

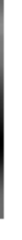

Fig. 3 BEMD applied to a part of a retina image: example

Following Nunes and al. [8], the bidimensional sifting process is defined as follows : 
- Identify the extrema (maxima and minima) of the image I.

- Generate the 2D 'envelope' by connecting maxima points (respectively, minima points) with 2D interploation methods.

- Averaging the two envelopes to compute the local mean $\mathrm{m}_{1}$.

- Since IMF should have zero local mean, subtract out the mean from the image: $\mathrm{I}-\mathrm{m}_{1}=\mathrm{h}_{1}$.

- repeat as $\mathrm{h}_{1}$ is an IMF.

In this paper we use the fast and adaptive BEMD presented in [11]. We give in Fig 3 an example of the BEMD for a retinal image.

\section{Numerical image characterization: signatures}

In this paper we suggest to characterize globally images by generating a numerical signature of image, based on IMFs contents. Wouwer and al. [9] show that, for textured images, the distribution of coefficients in the subbands of the wavelet transform may be modeled by a generalized gaussian law. Empirically, we noticed that the distribution of coefficients derived from IMFs seems also to follow a generalized gaussian law as illustrated below.
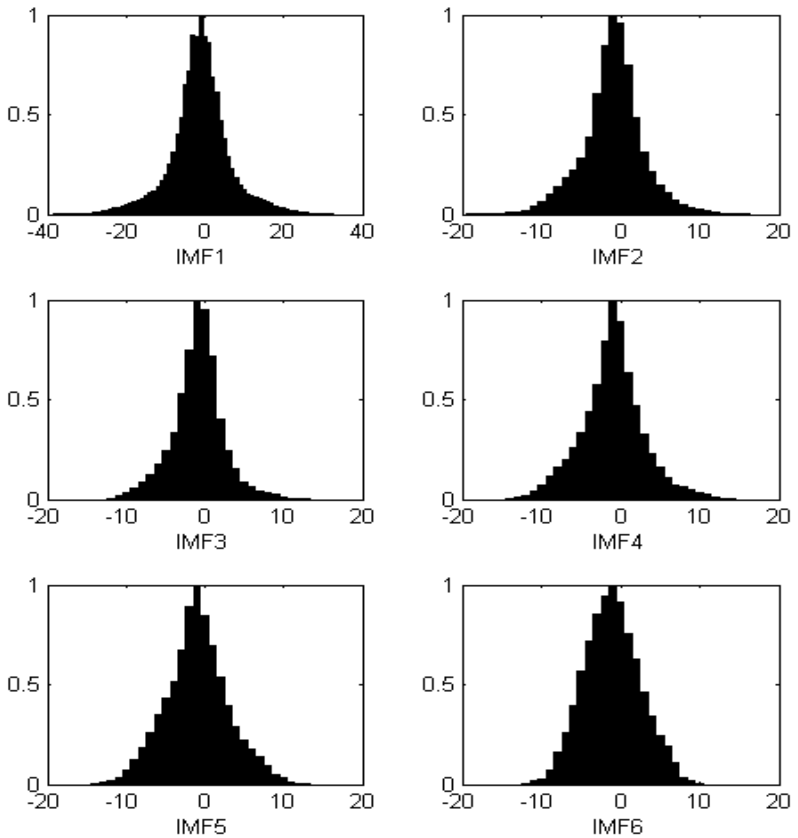

Fig. 4 BEMD IMFs coefficients histogram example for the image decomposed in Fig 3 .

The gaussian generalized law is derived from the normal law and parameterized by:
- $\alpha$ : a scale factor, it corresponds to the standard deviation of the classical Gaussian law.

- $\beta$ : a shape parameter.

So the law density is defined as.

$$
P(x ; \alpha, \beta)=\frac{\beta}{2 \alpha \Gamma\left(\frac{1}{\beta}\right)} e^{-\left(\frac{|x|}{\alpha}\right)^{\beta}}
$$

Where $\Gamma\left(\right.$.) is the gamma function, $\Gamma(z)=\int_{0}^{\infty} e^{-t} t^{z-t} d t, z>0$

We propose to characterize images by using a maximum likelihood estimator $(\hat{\alpha}, \hat{\beta})$ of the distribution law for coefficients of each IMF of the BEMD decomposition [4]. These estimators are defined like this: given $\mathrm{x}=\left(x_{1}, \ldots, x_{L}\right)$ the BEMD coefficients for one IMF. As $\mathrm{x}$ having independent component, Varanasi and Aazhang [12] demonstrated that $(\hat{\alpha}, \hat{\beta})$ is the unique solution of equation (3).

$$
\begin{array}{r}
\hat{\alpha}=\left(\frac{\hat{\beta}}{L} \sum_{i=1}^{L}\left|x_{i}\right|^{\hat{\beta}}\right)^{\frac{1}{\beta}} \\
1+\frac{\psi\left(\frac{1}{\hat{\beta}}\right)}{\hat{\beta}}-\frac{\sum_{i=1}^{L}\left|x_{i}\right|^{\hat{\beta}} \log \left|x_{i}\right|}{\sum_{i=1}^{L}\left|x_{i}\right|^{\hat{\beta}}}+\frac{\log \left(\frac{\hat{\beta}}{L} \sum_{i=1}^{L}\left|x_{i}\right|^{\hat{\beta}}\right)}{\hat{\beta}}=0
\end{array}
$$

where $\psi($.$) is the digamma function \psi(z)=\frac{\Gamma^{\prime}(z)}{\Gamma(z)}$.

$\hat{\beta}$ value should be find first by a Newton-Raphson algorithm. If the Newton-Raphson iterative algorithm is well initialized, it converges with few iterations.

We defined the image vector signature by the couple $(\hat{\alpha}, \hat{\beta})$ derived from each IMF.

\section{A. Distance}

To compute the similarity distance between to IMFs (Generalized Gaussian) and, according to [13] KullbackLeibler distance is used (see equation (5)).

$K L\left(p\left(X ; \theta_{q}\right) \| p\left(X ; \theta_{i}\right)\right)=\int p\left(X ; \theta_{q}\right) \log \frac{p\left(X ; \theta_{q}\right)}{p\left(X ; \theta_{i}\right)} d x$

In the case of generalized gaussian distributions, we inject equation (3) in equation (5), to obtain the following distance (equation (6)):

$K L\left(p\left(X ; \alpha_{1}, \beta_{1}\right) \| p\left(X ; \alpha_{2}, \beta_{2}\right)\right)=\log \left(\frac{\beta_{1} \alpha_{2} \Gamma\left(\frac{1}{\beta_{2}}\right)}{\beta_{2} \alpha_{1} \Gamma\left(\frac{1}{\beta_{1}}\right)}\right)+\left(\frac{\alpha_{1}}{\alpha_{2}}\right)^{\beta_{2}} \frac{\Gamma\left(\frac{\beta_{2}+1}{\beta_{1}}\right)}{\Gamma\left(\frac{1}{\beta_{1}}\right)}-\frac{1}{\beta_{1}}$ 
The distance between two images $I_{1}$ and $I_{2}$ is the sum of the balanced distance between IMFs.

$D\left(I_{1}, I_{2}\right)=\sum_{k=1}^{K} \lambda_{k} K L D\left(p\left(X, \alpha_{1}^{K}, \beta_{1}^{K}\right), p\left(X, \alpha_{2}{ }^{K}, \beta_{2}{ }^{K}\right)\right)$

where $\lambda_{k}$ are the adjustment weights.

\section{RESULT}

The method is tested on the two databases (see II-A), and is compared with previous methods detailed in $[4,5]$.

The evaluation criteria is the mean precision at 5 , which is the ratio between the number of pertinent images recalled and the total images recalled (here 5).

1) Each image in the database is used as a query image.

2) The algorithm find the five first images of the database closest to the query image.

3) Precision is computed for this query.

4) Finally, we compute the mean precision.

\begin{tabular}{llll}
\hline Database & $\begin{array}{l}\text { Number of IMFs } \\
\text { extracted for } \\
\text { each image }\end{array}$ & $\begin{array}{l}\text { BEMD- } \\
\text { GG }\end{array}$ & BEMD-Gabor \\
\hline Faces & 4 & $85.55 \%$ & $89.75 \%$ \\
Retinas & 6 & $43.75 \%$ & $52.40 \%$ \\
\hline & & \\
\hline Database & Decomposition level number & $\begin{array}{l}\text { Adapted wave- } \\
\text { lets-GG }\end{array}$ \\
\hline Faces & 2 & $95.50 \%$ \\
Retinas & 3 & $46.10 \%$ \\
\hline
\end{tabular}

Table 1 Mean precision at 5 .

From Table 1 we can see that a method based on adapted wavelets [14] achieved the highest means precision $95.5 \%$ for face database with Adapted wavelets-GG, and $52.40 \%$ for retinas database with BEMD-Gabor method.

Extracting all IMFs of retinas database takes 1 day, and few minutes for face database. Then query is very fast. For this experiment we used $\mathrm{C}++$ language on a AMD Athlon $64 \mathrm{X} 2$ dual Core processor working on $2 \mathrm{GHz}$ with $2 \mathrm{~Gb}$ DRAM.

\section{CONCLUSION}

In this paper, we proposed a new method to characterize the numerical content of medical images, where BEMD coefficients in each IMF are independently modeled by a generalized Gaussian density law (GGD). To compute the measure of similarity between GGDs, we used the Kullback-Leibler Distance (KLD). BEMD-GGD functions pro- posed in this paper achieved encouraging experimental results.

For that purpose, our future work is to try to enhance retrieval results, by, first, optimizing adjustment weights (genetic algorithms). And second, by fusing of semantic (clinical data) and image numerical content.

\section{REFERENCES}

1. C. Nastar, "Indexation d'images par le contenu: un etat de l'art," in CORESA'97, March 1997.

2. A. Smeulders, M.Worring, S. Santini, A. Gupta, and R. Jain, "Contentbased image retrieval at the end of the early years," IEEE Transactions on Pattern Analysis and Machine Intelligence, vol. 22, no. 12, pp. 1349-1380, December 2000.

3. M. Lamard, W. Daccache, G. Cazuguel, C. Roux, and B. Cochener, "Use of jpeg-2000 wavelet compression scheme for content-based ophtalmologic retinal retrieval," in Proceedings of the 27th annual international conference of IEEE engeneering in medecine and biology society, september 2005.

4. M. Lamard, G. Cazuguel, G. Quellec, L. Bekri, C. Roux, B. Cochener, "Content Based Image Retrieval based on Wavelet Transform coefficients distribution" in Proceedings of the 29th Annual International Conference of the IEEE EMBS, Lyon, France August 23-26, 2007.

5. S. Jai-Andaloussi, M. Lamard, G. Cazuguel, H. Tairi, M. Meknassi, B. Cochener, C. Roux, "Recherche d'images médicales par leur contenu numérique : utilisation de signatures construites à partir de la bemd "Journées de Recherche en Imagerie et Technologies de la Santé, Lille, France Mars 17-20, 2009.

6. B.S. Manjunath, P. Wu, S. Newsam, and H.D. Shin. "A texture descriptor for browsing and similarity retrieval". Journal of Signal Processing: Image Communication, 16(1-2) :33-43, 2000.

7. Huang and al, "The empirical mode decomposition and the Hilbert spectrum for non linear and non-stationary time series analysis," Proc. Roy. Soc. London A, Vol. 454, pp. 903-995, 1998.

8. J. C. Nunes, Y. Bouaoune, E. Delechelle, O. Niang, Ph. Bunel, "Image analysis by bidimensional empirical mode decomposition," Image and Vision Computing, Vol. 21 pp. 1019-1026, 2003.

9. G. V. Wouwer, P. Scheunders, and D. V. Dyck, "Statistical texture characterization from discrete wavelet representations," IEEE Trans. Image Processing, vol. 8, pp. 592-598, Apr. 1999.

10. F. Samaria and A. Harter, "Parameterisation of a stochastic model for human face identification," in 2nd IEEE Workshop on Applications of Computer Vision, Sarasota FL, december 1994.

11. Bhuiyan, S.M.A, Adhami, R.R. Khan, J.F, "A novel approach of fast and adaptive bidimensional empirical mode decomposition" March 31 2008-April 42008 ICASSP 2008. IEEE International Conference.

12. M. Varanasi and B. Aazhang, "Parametric generalized gaussian density estimation" J. Acoust. Soc. Amer., vol. 86, pp. 1404-1415, 1989.

13. M.N. Do, M.Vetterli," Wavelet-Based Texture Retrieval Using Generalized Gaussian Density and Kullback-Leibler Distance" IEEE TRANSACTIONS ON IMAGE PROCESSING, VOL. 11, NO. 2, February 2002

14. G. Quellec, M. Lamard, P.M. Josselin, G. Cazuguel, B. Cochener, C. Roux "Optimal wavelet transform for the detection of microaneurysms in retina photographs", IEEE Transactions on Medical Imaging (September 2008, Vol. 27, N 9, p 1230-1241) 